\title{
AUFSÄTZE
}

\section{Non-formale Bildung in nationalen Freiwilligendiensten - Zwischen beruflicher Orientierung und kritisch-emanzipatorischem Anspruch ${ }^{1}$}

\author{
Stefanie Bonus \\ M.A. Soziale Arbeit | Technische Hochschule Köln \\ (Forschungsschwerpunkt Nonformale Bildung) \\ stefanie.bonus@th-koeln.de \\ Stefan Schäfer \\ M.A. Soziale Arbeit | Technische Hochschule Köln \\ (Fakultät für Angewandte Sozialwissenschaften) \\ stefan.schaefer@th-koeln.de \\ Stefanie Vogt \\ Dipl. SozPäd | M.A. Soziale Arbeit | Technische Hochschule Köln \\ (Fakultät für Angewandte Sozialwissenschaften) \\ stefanie.vogt@th-koeln.de
}

\section{Zusammenfassung}

Dass Freiwilligendienste eine Vielfalt an Bildungsmöglichkeiten bieten, scheint unbestritten und stellt gleichzeitig keine Selbstverständlichkeit dar. Bildung in Freiwilligendiensten bewegt sich in einem Spannungsfeld zwischen kritisch-emanzipatorischem Anspruch und einer Funktionalisierung von Bildung im Kontext ökonomischer und gesellschaftlicher Anforderungen der Arbeits- und Wissensgesellschaft. Der Beitrag stellt ausgewählte Ergebnisse eines Praxisentwicklungs- und Praxisforschungsprojekts vor, das in Kooperation zwischen Forschung und Praxis durchgeführt wurde. Non-formale Bildung wird hierbei im Sinne einer Eigenaktivität der Freiwilligen verstanden. Subjektorientierung und Partizipation werden als zentrale Arbeitsprinzipien ins Zentrum der Bildungsarbeit in den Freiwilligendiensten gerückt. Der Beitrag zeigt über die ideelle Idee einer kritischemanzipatorischen Bildung hinaus konzeptionelle Ansatzpunkte für die pädagogische Praxis auf.

Schlagwörter: non-formale Bildung, Freiwilligendienst, Partizipation, Subjektorientierung

1 Der Artikel basiert auf Forschungsergebnissen im Projekt „Non-formale Bildung in den Inlandsfreiwilligendiensten“, welches durch den Forschungsschwerpunkt Non-formale Bildung der TH Köln im Auftrag des Internationalen Bundes von April 2015 bis März 2017 durchgeführt wurde. Grundlage des vorliegenden Aufsatzes ist der Abschlussbericht der wissenschaftlichen Begleitung des Projekts (Bonus/Vogt 2018), der beispielhaft skizziert, wie konzeptionelle Themenbereiche und Bildungsziele sowie Arbeitshilfen entwickelt werden können. 


\begin{abstract}
The notion that voluntary services offer a variety of educational opportunities, seems undisputed and represents at the same time not a matter of course. Education in voluntary services moves in a field of tension between critical emancipatory claim and a functionalization of education, in the context of economic and social needs of the labour market requirements and knowledge-based society. The article presents selected results of a development-and practice research project, which was carried out in cooperation between research and practice. Non-formal education is understood in the sense of a self-activity of the volunteers. Subject-orientation and participation are the central working principles at the center of educational work in voluntary services. The article shows conceptual ideas for pedagogical practice beyond the ideal idea of a critical-emancipatory education.

Keywords: non-formal education, voluntary service, participation, subject-orientation
\end{abstract}

\title{
1. Einleitung
}

Seit einiger Zeit wird in der Praxis und den wissenschaftlichen Diskursen um Freiwilligendienste ${ }^{2}$ verstärkt dafür plädiert, diese als Bildungsjahr zu verstehen (u. a. Thimmel/Brombach 2018; Brombach 2014; Jakob 2014; Olk/Klein 2014; Rauschenbach 2015). Sowohl in den Gesetzen zu den unterschiedlichen Formaten des Freiwilligendienstes als auch in den Fachdiskursen wird impliziert, dass Freiwilligendienste insbesondere durch die Verbindung von sozialem Engagement in Einsatzstellen und pädagogischen Begleitseminaren vielfältige Bildungsmöglichkeiten bieten. Laut dem Jugendfreiwilligendienstgesetz (JFDG), welches 2008 in Kraft trat und aus dem Gesetz zur Förderung eines freiwilligen sozialen Jahres entstanden ist, fördert der Freiwilligendienst die „Bildungsfähigkeit von Jugendlichen“ (JFDG §1 Abs. 1). Im Bundesfreiwilligendienstgesetz (BFDG) ist die Rede von der Förderung des „lebenslangen Lernens“ (BFDG §1 Abs. 1). In $§ 4$ Abs. 1 des BFDG heißt es zudem, dass der „Bundesfreiwilligendienst [...] pädagogisch begleitet [wird] mit dem Ziel, soziale, ökologische, kulturelle und interkulturelle Kompetenzen zu vermitteln und das Verantwortungsbewusstsein für das Gemeinwohl zu stärken“. Wird der Jugendfreiwilligendienst als Teil der Kinder- und Jugendhilfe verstanden (Slüter 2008: 17), zielt der Bildungsauftrag entsprechend §11 SGB VIII auf allgemeine, politische, soziale, gesundheitliche, kulturelle, naturkundliche und technische Bildung (Nugel u. a. 2014: 10).

Gleichzeitig zeigt sich, dass im Fachdiskurs unterschiedliche Bildungsverständnisse bestehen. Die uneinheitliche Verwendung der Begriffe formale Bildung, non-formale Bildung, informelle Bildung, informelles Lernen, nicht-formale Bildung

2 Sofern nicht anders beschrieben, bezieht sich der Begriff Freiwilligendienste in diesem Beitrag in erster Linie auf die Inlandsfreiwilligendienste Freiwilliges Soziales Jahr (FSJ), Freiwilliges Ökologisches Jahr (FÖJ) und den Bundesfreiwilligendienst (BFD). 
oder auch informelles Lernen im Non-formalen geht mit unterschiedlichen und teils widerstreitenden Begriffsverständnissen einher. Für Olk und Klein (2014: 20) stellt der Jugendfreiwilligendienst „einen non-formalen bzw. informellen Lernort dar, an dem junge Menschen wichtige Kompetenzen erwerben können“. Nach Fischer (2011: 58) sind Freiwilligendienste Lernorte der nicht-formalen Bildung, in denen „sich Lernen (und auch Wirkung) als Veränderung von Wissen, Verhalten und Einstellung" manifestiert. Dieser allgemeine Bildungsanspruch wird unter anderem in den entsprechenden Bundesgesetzen mit funktionalen Zielsetzungen verknüpft, indem es beispielsweise darum geht, die „Förderung der Bildungs- und Beschäftigungsfähigkeit der Freiwilligen“( JFDG §5 Abs. 4) und/oder „berufsqualifizierende Merkmale des Bundesfreiwilligendienstes“ (BFDG §1 Abs. 2) in das schriftlich zu erstellende Zeugnis der Einsatzstellen aufzunehmen. Diese Funktionalisierung und Verzweckung von Bildung im Sinne einer arbeitsmarktpolitischen Maßnahme widerspricht einem emanzipatorischen Verständnis von Bildung als Selbstbildung (Scherr 1997; Münchmeier/Otto/Rabe-Kleberg 2002). Die Idee von Bildung als Selbstbildung positioniert sich gegen die Vorstellung, dass Bildung von einer außenstehenden Kraft induziert werden könne. Zentral ist damit ein subjektbezogenes und emanzipatorisches Bildungsverständnis als Grundlage der Bildungsarbeit in Freiwilligendiensten. Insofern bewegt sich die Betonung des Bildungsaspekts in den Freiwilligendiensten in einem Spannungsverhältnis zwischen kritisch-emanzipatorischem Anspruch und Funktionalisierung (vgl. auch Roth 2017).

Freiwilligendienste stellen mit ihren Rahmenbedingungen ein spezifisches Setting der non-formalen Bildung dar, welches sich von anderen Feldern der nonformalen Bildung, etwa der Jugendverbandsarbeit oder der Offenen Kinder- und Jugendarbeit unterscheidet (Thimmel 2016: 21). Um die Besonderheit non-formaler Bildungsorte zu verstehen, werden wir im Folgenden eine systematische Differenzierung von formaler, informeller und non-formaler Bildung vornehmen. Anschließend geben wir einen kurzen Einblick in Ergebnisse eines zweijährigen Forschungsprozesses, welcher zum Ziel hatte, den Zusammenhang von non-formaler Bildung und Freiwilligendiensten zu verstehen und aufzuzeigen, wie nonformale Bildungsarbeit im Bereich der Freiwilligendienste konkret wird. Hier wurde versucht, gelebte Bildungspraxis mit qualitativen Methoden der Sozialforschung sichtbar zu machen. Dabei haben sich drei verschiedene Typen der Bildungsarbeit herauskristallisiert, die im dritten Teil beschrieben werden. Ausgehend davon werden wir abschließend Kriterien non-formaler Bildungsarbeit skizzieren, die Hinweise auf eine weitere konzeptionelle Ausgestaltung von Freiwilligendiensten als Bildungsjahr geben und dabei Subjektorientierung und Partizipation als Arbeitsprinzipien ins Zentrum rücken. 


\section{Systematik non-formaler Bildungsräume}

Der Bildungsbegriff wird im pädagogischen Diskurs vieldeutig gebraucht. Aus dem europäischen Diskurs um das Konzept des Lebenslangen Lernens wurde in der deutschen Fachdebatte die Differenzierung zwischen formaler Bildung, informellem Lernen und non-formaler Bildung eingeführt (Rauschenbach u. a. 2004). Diese Unterscheidung von Bildungsorten verbindet die erziehungswissenschaftliche Erforschung von Bildungsprozessen mit der Frage nach unterschiedlichen institutionellen Rahmenbedingungen, Organisationsgraden und pädagogischen Prinzipien. Allerdings ergibt erst deren Zusammenspiel Bildung im umfassenden Sinn (Münchmeier/Otto/Rabe-Kleberg 2002: 164 f.). Die diskutierten Verständnisweisen von formaler Bildung, informellem Lernen und non-formaler Bildung lassen sich wie folgt zusammenfassen:

Formale Bildung „bezieht sich auf das gesamte, hierarchisch strukturierte und zeitlich-biographisch aufeinander aufbauende Schul-, Ausbildungs- und Hochschulsystem" (Thimmel 2017: 226). Als formaler Bildungsort ist im Kindes- und Jugendalter insbesondere die Schule in ihren unterschiedlichen Schulformen zentral. Lernen ist an formalen Bildungsorten stark strukturiert, geplant, reguliert und verpflichtend. In formalisierten Bildungssettings werden meistens altershomogene Gruppen in überwiegend standardisierten Räumlichkeiten und in festgelegten Zeitfenstern unterrichtet. Die Inhalte orientieren sich an den jeweiligen Lehrplänen und Curricula. Lernfortschritte und Kompetenzen werden in Form von Lernstandserhebungen gemessen, vergleichbar gemacht und in Zertifikaten und Qualifikationen dokumentiert, wodurch wiederum Zugänge zu anderen Institutionen ermöglicht und begrenzt werden. Das formale Bildungssystem übernimmt damit eine zentrale Rolle in der gesellschaftlichen Verteilung von Lebenschancen (Thimmel 2017: 226).

Als informelles Lernen, teils auch als informelle Bildung bezeichnet, werden ungeplante und nicht beabsichtigte Lern- und Bildungsprozesse verstanden, die sich im Alltag und aus den alltäglichen Handlungsvollzügen heraus ergeben. Als informelle Bildungsorte gelten insbesondere die Familie, die Nachbarschaft, die Gruppe der Gleichaltrigen sowie Medien (Rauschenbach u. a. 2007). Informelles Lernen bezeichnet damit alle Formen des selbstbestimmten Lernens, die selbstverständlich auch in pädagogisch gerahmten formalen wie non-formalen Bildungsorten geschehen. Das entscheidende Merkmal besteht aber darin, dass die pädagogische Rahmung des Lernsettings nicht ausschlaggebend dafür ist, womit sich beschäftigt wird und wie die jeweilige Beschäftigung aussehen soll. Mit Faulstich (2013: 132), der hier von „menschlichem Lernen“ spricht, kann zusammenfassend gesagt werden, dass informelles Lernen immer dann stattfindet, wenn der Lernende in der alltäglichen Interaktion mit Anderen mit Brüchen, Irritationen und Zweifeln in der eigenen Handlungsroutine konfrontiert wird, die auf 
zu Bewältigendes verweisen und darum der Intentionalität der Lernenden eine bestimmte Richtung geben. Informelles Lernen ist damit als Umformung von in der alltäglichen Auseinandersetzung mit der natürlichen, kulturellen, sozialen und politischen Welt resultierenden Handlungsaufforderungen in individuelle Lernstrategien zu verstehen, die das Aktivitätsspektrum der Subjekte erweitern und/ oder modifizieren (Thimmel 2017: 226).

Non-formale Bildung bezeichnet Formen organisierter Bildung, Freizeitgestaltung und Ermöglichung von Gelegenheitsstrukturen, die in der Regel freiwilliger Natur sind, jedoch im Unterschied zum informellen Lernen die Anwesenheit von Pädagog*innen voraussetzen oder zumindest als im weitesten Sinne pädagogisch gerahmtes Setting anzusehen sind. Non-formale Bildungsorte bieten unterschiedlich stark strukturierte Räume, in denen Lernen intendiert ist, es aber keinen Lehrplan und keine Lernstandserhebungen gibt und im Allgemeinen auch keine Zertifikate ausgestellt werden. Die Differenziertheit non-formaler Bildungsorte reicht von der Offenen Kinder- und Jugendarbeit, über die Jugendbildungsarbeit, die Jugendsozialarbeit bis zur Weiterbildung. Die hier stattfindenden Bildungsprozesse schließen den Erwerb von Kompetenzen selbstverständlich mit ein, Bildungsprozesse lassen sich aber nicht auf Kompetenzerwerb reduzieren. In diesem Sinne lässt sich non-formale Bildung als subjektorientierte Bildung verstehen, die an den Erfahrungen, Bedürfnissen und Interessen ihrer Adressat*innen ansetzt. Dies impliziert eine traditionell emanzipatorisch orientierte Methodik, deren Ausgangspunkt die Lebenswelt der Adressat*innen ist und auf Selbstbestimmung, gesellschaftliche Mitverantwortung und gesellschaftliches Engagement zielt. Hieraus ergibt sich ein politischer Bildungsanspruch non-formaler Bildung, der auf die Entwicklung von Urteilskraft, Reflexion und Handlungsfähigkeit in sozialen Beziehungen und gesellschaftlichen Verhältnissen zielt. Die Anerkennung von Verschiedenheit sowie die Berücksichtigung der Eigensinnigkeit der Adressat*innen zählen zu den Bedingungen, um Handlungsfähigkeiten und Bewältigungsstrategien zu erweitern. Als Teil eines demokratischen und gesellschaftspolitischen Auftrags verstehen sich die Handlungsfelder non-formaler Bildung als Beitrag, Menschen darin zu fördern, Politik und Gesellschaft kritisch zu begleiten und mitzugestalten. Die konstitutiven Prinzipien der Freiwilligkeit, Offenheit und Partizipation sowie die Orientierung an den alltäglichen Erfahrungen, Bedürfnissen und Interessen prägen die Ausgestaltung non-formaler Bildungsorte. Dieser fachlich verantwortete Rahmen zielt auf Ermöglichung und Unterstützung von selbsttätiger Bildung im sozialpädagogischen Sinne, das heißt durch aktive Unterstützung, durch Gelegenheiten, Anlässe und Aufgaben, durch Begleitung, Aufklärung und Reflexion. Die Stärke non-formaler Bildungsorte besteht somit darin, der Breite von Lebensthemen gerecht zu werden. Damit sind Freizeit und zweckfreie Kommunikation, Spaß und Geselligkeit selbstverständliche Bestandteile eines reflektierten Konzepts non-formaler Bildung. Alltagsbezug, informelles Lernen, thematische 
Angebote, Erfahrungen in der Gruppe, Erlebnisse und individuelle Herausforderungen in der Lebensbewältigung gehen dabei ein konstruktives Mischungsverhältnis ein (Thimmel 2017: 226 f.).

\section{Forschungsfrage, Forschungsmethoden und Typenbildung}

In unseren Forschungen haben wir den Fokus auf die Bildungsarbeit in den Begleitseminaren gelegt und nach dem Bildungsverständnis der pädagogischen Fachkräfte sowie der pädagogischen Gestaltung der Seminararbeit gefragt. Zentrale Fragestellungen des Praxisforschungsprojekts lauteten: Was verstehen die pädagogischen Fachkräfte unter Bildung in Freiwilligendiensten? Wie wird die Bildungsarbeit in den Begleitseminaren gestaltet?

Datengrundlage bildeten zehn Expert*inneninterviews mit pädagogischen Fachkräften und teilnehmende Beobachtungen bei Bildungsseminaren. Über die Expert*inneninterviews wurde das subjektive Deutungs- und Handlungswissen erforscht, welches dann diskutiert und konzeptionell abgebildet wurde (Bonus/ Vogt 2018: 20). Das Ziel der qualitativen Interviews bestand darin, etwas über die Themen zu erfahren, welche die Fachkräfte in Bezug auf ihre Bildungspraxis bewegen. Analysiert wurde das Datenmaterial der Expert*inneninterviews mithilfe des inhaltsanalytischen Vorgehens nach Meuser und Nagel (1991). Das Datenmaterial wurde transkribiert, paraphrasiert, kategorisiert, thematisch verglichen und anschließend theoretisch konzeptualisiert und abschließend typologisiert.

Zudem wurden in drei Bildungsseminaren teilnehmende Beobachtungen durchgeführt. Das Ziel der Beobachtungen bestand in der Analyse der Umsetzungspraxis non-formaler Bildungsarbeit durch die Träger. Hierbei stand die Beobachtung sozialer Praktiken im Mittelpunkt, die Aufschluss darüber geben, wie sich non-formale Bildungsarbeit als soziale Praxis konkret ausgestaltet. Der Beobachtungsfokus wurde auf die Ermöglichung und Begrenzung von Aushandlungsprozessen gelegt. Die Beobachtungen wurden anhand von narrativen Beobachtungsprotokollen dokumentiert und in einer Kombination aus inhaltsanalytischem Vorgehen und Grounded-Theory-Methodologie ausgewertet (Cloos 2010: 21).

Die verschiedenen Bildungsverständnisse und Bildungspraxen (nicht Bildungsprozesse) der befragten Fachkräfte wurden u. a. in drei exemplarischen ,Bildungstypen' beschrieben. Benannt wurden diese als: ,Fit machen und Orientierung' (Typ I), ,Vermittlung und soziales Lernen' (Typ II) und ,Ermöglichung und Selbstbestimmung' (Typ III). Die Typenbildung ist aus forschungsmethodischer Sicht ein Gruppierungsprozess, bei dem die gebildeten Gruppen als „Typen“ bezeichnet werden, die sich durch eine jeweils charakteristische Anordnung verschiedener Merkmalsausprägungen und Eigenschaften auszeichnen und beschreiben lassen (vgl. Kelle/Kluge 2010: 85). Es geht dabei „um die Herausarbeitung 
gegenstandsbezogener Systematisierungen, die für Beschreibungs-, Erklärungsund Selbst- bzw. Handlungsreflexionszwecke tauglich" sind (Breuer 2009: 90). Die herausgearbeiteten Typen bieten eine Interpretationshilfe für die Analyse der verschiedenen Bildungsverständnisse und Bildungspraxen. Allerdings ist zu beachten, dass die Typen eine heuristisch-theoretische Konstruktion darstellen, ohne eine deckungsgleiche Entsprechung in der empirischen Wirklichkeit zu haben (vgl. Schmidt-Hertha/Tippelt 2011: 25). Das heißt, bei jedem der erhobenen Einzelfälle überschneiden und überlagern sich die verschiedenen Typen in je spezifischer Ausprägung (vgl. Nentwig-Gesemann 2013: 317) und die Bildungstypen sind losgelöst von einzelnen Personen und Standorten zu betrachten. (vgl. Bonus/ Vogt 2018: 43)

\section{Drei Typen non-formaler Bildungsarbeit im Freiwilligendienst}

Die Besonderheit von Freiwilligendiensten kann „im Zusammenspiel von Bildungsprozess und Orientierungsphase einerseits und der Übernahme sozialer Verantwortung und Tätigkeit mit ,Ernstcharakter" andererseits“" (Jakob 2011: 186) gesehen werden. Die Freiwilligen werden im Rahmen der begleitenden Seminare und den Einsatzstellen in verantwortungsvolle Tätigkeiten eingebunden und erhalten die Möglichkeit, vielfältige Wissensbestände und Fertigkeiten zu erwerben und ihre Erfahrungen zu reflektieren. Ein Teil der pädagogischen Begleitung besteht aus gesetzlich vorgeschriebenen Bildungsseminaren.

Der folgende Abschnitt gibt einen Einblick in die Vielfalt der non-formalen Bildungsarbeit im Kontext von Freiwilligendiensten anhand der drei generierten Bildungstypen.

Der erste Bildungstyp, den wir mit Fit machen und Orientierung überschrieben haben, ist charakterisiert durch ein weitgehend funktionales Bildungsverständnis. Die non-formale Bildungsarbeit in den Freiwilligendiensten kennzeichnet sich für diesen Bildungstyp vor allem durch eine Orientierungs- und Vorbereitungsfunktion. Die Gestaltung der Bildungsangebote orientiert sich an den Bewältigungsanforderungen, die an die Freiwilligen im Rahmen ihrer Tätigkeit in den Einsatzstellen gestellt werden. Inhalte und Ziele der Bildungsarbeit richten sich an fachlichen und beruflichen Anforderungen aus und verknüpfen sich hierüber mit einer Nutzenorientierung. Die Auswahl der Inhalte und Themen wird einerseits begründet mit dem praktischen Nutzen für die Tätigkeit in den Einsatzstellen (Nutzen für jetzt) und andererseits mit dem Nutzen für den Zugang zum Ausbildungs- und Arbeitsmarkt (Nutzen für später). Ziel dieses Typs ist es, die Freiwilligen für die Arbeit in den Einsatzstellen sowie den Ausbildungs- und Arbeitsmarkt „fit zu machen“, indem eine berufliche Orientierung ermöglicht und die Einsatz- und Ausbildungsfähigkeit der Freiwilligen gefördert werden. Vor diesem 
Hintergrund wird insbesondere die Vermittlung von fachspezifischen Wissensbeständen und Kompetenzen sowie von Werten und Normen zentraler Bestandteil der Seminararbeit. Das Seminarsetting ist eher geschlossen organisiert und bietet wenige Partizipationsmöglichkeiten. Relevante Themen im Hinblick auf die Einsatzstellen und die Berufsorientierung werden teilweise im Dialog mit den Freiwilligen und im Rahmen der Reflexion von Erfahrungen gemeinsam hergestellt. Entsprechende Angebote werden in einem Prozess entwickelt, der große Flexibilität von den pädagogischen Fachkräften erfordert. Daneben wird versucht, im Vorfeld zu antizipieren, welche Wissensbestände und Kompetenzen die Freiwilligen benötigen. Die Gefahr dieses Typs besteht darin, Bildung im Sinne umfassender Persönlichkeitsbildung einschließlich gesellschaftspolitischer Bildung zu vernachlässigen und entlang realer oder antizipierter Bewältigungsanforderungen zu verengen. Die Seminararbeit ist dann davon geprägt, dass die pädagogischen Fachkräfte die Inhalte und Formen von Bildung steuern und definieren. Themen, Interessen und das Handeln der Freiwilligen werden zwar aufgegriffen, aber in eine bestimmte Richtung geführt. Die Lernarrangements sind sowohl inhaltlich als auch methodisch entsprechend stark vorstrukturiert. Die pädagogischen Fachkräfte erscheinen hierbei eher als Lehrende, Dozierende und/oder Erziehende und weniger als Bildungsbegleiter*innen.

Davon zu unterscheiden ist der zweite Bildungstyp Vermittlung und soziales Lernen. Zentraler Bestandteil der Seminararbeit dieses Typs sind Wissensvermittlung und der Erwerb sozialer Kompetenzen. Hierbei wird Bildung als Aneignung gesellschaftlich anerkannter Verhaltensweisen und Wissensbestände verstanden. Als Ziele werden die Vermittlung sozialer Kompetenzen und Verhaltensweisen wie gewaltfreie Konfliktlösung, Empathie und Toleranz genannt. Diese Zielsetzungen werden begründet, indem soziale Kompetenzen als Grundlage für das gelingende Zusammenleben in einer heterogenen Gesellschaft und eine erfolgreiche Arbeit in den Einsatzstellen betrachtet werden. Wahrgenommene Wissens- und Verhaltensdefizite und Verhaltensabweichungen der Freiwilligen sollen vor diesem Hintergrund bearbeitet oder verhindert werden. Non-formaler Bildung im Rahmen der Freiwilligendienste wird dabei auch eine kompensatorische Funktion gegenüber anderen Sozialisationsinstanzen wie zum Beispiel der Schule und der Familie zugeschrieben. So wird beispielsweise davon ausgegangen, dass in der Schule nicht vermittelte soziale Kompetenzen in den Freiwilligendiensten erworben werden können. Freiwilligkeit, Partizipation und Offenheit werden zugunsten von Regelungen, stärkerer Strukturierung, Konsequenzen und gegebenenfalls Sanktionen begrenzt. Partizipation wird insbesondere als Wahlfreiheit hinsichtlich zu bearbeitender Themen und Inhalte verstanden. Die Vermittlung bestimmter Wissensbestände, die aus Sicht der Seminarleitung eine themenbezogene Relevanz besitzen, sowie das Erlernen bestimmter, allgemein anerkannter sozialer Verhaltensweisen und Kompetenzen sind Bestandteil jeder Form von non-formaler 
Bildung. Die Schwierigkeit dieses Bildungstyps besteht darin, dass Wissensbestände und bestimmte Verhaltensweisen durch die Seminarleitung unreflektiert zum Gegenstand des Lernens gemacht werden, ohne dass eine kritische Auseinandersetzung mit den hierin eingelagerten Normalitätsvorstellungen garantiert ist.

Der dritte Bildungstyp Ermöglichung und Selbstbestimmung ist charakterisiert durch ein kritisch-emanzipatorisches Bildungsverständnis. Ausgangspunkt dieser Orientierung ist der Versuch der Ermöglichung gleicher Teilnahmeoptionen und die Unterstellung einer generellen Teilnahmemündigkeit. Hiermit wird die Autonomie- und Partizipationsfähigkeit der Freiwilligen anerkannt und die Freiwilligen werden als Dialogpartner*innen gesehen. Grenzen der eigenen fachlichen Expertise und Anleitung werden transparent gemacht und in der Interaktion mit Freiwilligen als solche markiert. Die Inhalte und Formen der Bildungsarbeit werden soweit wie möglich offen gehalten und im Prozess mit den Freiwilligen dialogisch bestimmt. Die Entwicklung von Reflexionsfähigkeit, Selbstbewusstsein, Wissen über eigene Fähigkeiten und Grenzen, Bedürfnisse und Interessen gehören ebenso zu den Zielen wie die Stärkung gesellschaftlicher Mitverantwortung und sozialen Engagements. Erfahrungen von Selbstwirksamkeit sollen insbesondere über die Realisierung eigener Projekte ermöglicht werden. Der starke Wunsch der Freiwilligen nach Aneignung fachlichen Wissens mit Bezug zur Tätigkeit in den Einsatzstellen kann eine Grenze dieses Bildungsverständnisses markieren und wird ambivalent bewertet. Einerseits wird die Vermittlung fachlichen Wissens von den befragten Fachkräften als Bestandteil der Seminararbeit beschrieben, sofern es dem Interesse der Freiwilligen entspricht. Anderseits wird dieser Wunsch der Freiwilligen nach thematischen Schwerpunkten in Bezug auf die Erfordernisse der Einsatzstellen als Ausdruck des gesellschaftlichen und beruflichen Leistungsund Anpassungsdrucks gewertet und kritisiert. In emanzipatorischer Absicht verstehen sie den Freiwilligendienst demgegenüber als Möglichkeit, die Freiwilligen zu einer kritischen Auseinandersetzung mit potenziellen und tatsächlichen Zwängen und Anforderungen der Arbeitswelt anzuregen. Die Ermöglichung von Selbstbestimmung ist das übergeordnete Bildungsziel dieses Typs. Gleichzeitig werden die unterschiedlichen sozialen Voraussetzungen und Begrenzungen von Selbstbestimmungsfähigkeiten der Freiwilligen berücksichtigt. So wird beispielsweise versucht, Freiwilligen mit Sprach-, Verständnis- und Verständigungsproblemen durch alternative Ausdruckformen wie Kunst und Musik Partizipationsmöglichkeiten zu eröffnen. Zu den Charakteristika dieses Typs zählt die Suche nach Freiräumen für experimentelles Handeln, innerhalb des stark strukturierten Settings Freiwilligendienst. Insbesondere die Seminararbeit wird als eine Möglichkeit gesehen, den Freiwilligen einen Raum zu eröffnen sich auszuprobieren, sich selbst zu reflektieren und eine Auszeit von den teils herausfordernden Aufgaben und Anforderungen in den Einsatzstellen zu ermöglichen. Freiwilligkeit wird als Freiheit und Offenheit für unterschiedliche Interessen und Formen von Engagement und 
Beteiligung verstanden. Partizipation wird soweit wie möglich herausgefordert, umgesetzt und in ihren Grenzen reflektiert. Gleichzeitig wird es für sinnvoll erachtet, den Freiwilligen so viel Sicherheit, Begleitung und Unterstützung wie notwendig anzubieten.

\section{Subjektorientierung und Partizipation als Arbeitsprinzipien non-formaler Bildungsarbeit in den Freiwilligendiensten}

Die Grenzen der einzelnen Bildungstypen sind fließend, auch wenn sich einzelne Bestandteile gegenseitig ausschließen. Als zentrale Kategorien eines typenübergreifenden non-formalen Bildungsverständnisses kristallisierten sich Subjektorientierung und Partizipation heraus. Auch wenn einzelne Bildungsarrangements und die artikulierten Ziele einem subjektorientierten und partizipatorischen Bildungsverständnis widersprechen, wurden Subjektorientierung und Partizipation zumindest dem Anspruch nach durchweg als übergeordnete Ziele und Arbeitsprinzipien formuliert. Sowohl in der Interpretation des Freiwilligendienstes als Bildungsjahr als auch in der Beschreibung der Bildungstypen lassen sich Subjektorientierung und Partizipation als grundlegende Arbeitsprinzipien nonformaler Bildungsarbeit identifizieren, wenngleich diese Zielsetzungen in der Bildungspraxis unterschiedlich stark verwirklicht werden. Daher möchten wir diese im Folgenden nochmals in den Blick nehmen und dabei auf die Grenzen in der pädagogischen Arbeit mit Freiwilligen verweisen.

Freiwillige werden dem Grundsatz der Subjektorientierung nach als Subjekte der Bildungsarbeit und nicht als zu Belehrende oder zu Erziehende verstanden. Die Anerkennung der Mündigkeit und Eigenständigkeit der Freiwilligen ist dabei zentral. Subjektorientierung verbindet sich mit dem übergeordneten Ziel, den Freiwilligen zu ermöglichen, im Handeln und Denken Selbstachtung, Selbstbewusstsein und Selbstbestimmung zu entfalten. Dies beinhaltet den Versuch der Etablierung symmetrischer Kommunikationsräume trotz bestehender Hierarchien und Machtasymmetrien. Gleichzeitig erscheint eine advokatorische Schutzfunktion und Grenzsetzung beispielsweise zur Sicherung der Arbeitsmarktneutralität und der Rechte der Freiwilligen gegenüber den Einsatzstellen notwendig. Subjektorientierung im Sinne eines emanzipatorischen Bildungsverständnisses schließt die Vermittlung von Wissen nicht aus, erschöpft sich aber auch nicht darin. Über seminaristische Bildungsangebote hinaus wird die Bildungsarbeit in den Freiwilligendiensten im Sinne einer Hilfe zur Lebensbewältigung durch Angebote der individuellen Beratung und Begleitung ergänzt. In diesem Sinne wird eine doppelte Perspektive vertreten, um den unterschiedlichen Erwartungen, die an die Freiwilligen gestellt werden, und der differenzierten pädagogischen Praxis gerecht zu werden. Der Ansatz der Subjektorientierung bietet die Möglichkeit, Bildung und Bewältigung zu verbinden und kann für Seminarleitung, Einsatzstellen und Träger 
als gemeinsamer Orientierungsrahmen dienen sowie unterschiedliche Schwerpunktsetzungen innerhalb der pädagogischen Praxis ermöglichen.

Partizipation kann im Sinne von Mitwirkung, Mitgestaltung und Mitbestimmung als eine Voraussetzung für den Erwerb sozialer und individueller Kompetenzen durch das Engagement im Freiwilligendienst angesehen werden. Freiwilligendienste fördern Partizipation, indem das Engagement der Freiwilligen als gesellschaftlich wichtiger Beitrag ernst genommen wird. Partizipation umfasst darüber hinaus die Berücksichtigung von Bedürfnissen und Interessen der Freiwilligen bezüglich der Arbeit in den Einsatzstellen und darüber hinaus die Möglichkeit der Mitbestimmung von Seminarthemen und die Mitgestaltung der Seminare. Zudem lässt sich Beteiligung im Kontext der Einsatzstelle, beispielsweise im Rahmen von Teamsitzungen, realisieren. Auch der Einbezug in die Öffentlichkeitsarbeit des Trägers sowie die Unterstützung der eigenständigen Vertretung und Organisation der Freiwilligen durch ein Sprecher*innensystem sind Beispiele, wie das Prinzip der Partizipation verwirklicht werden kann. Zugleich müssen die Grenzen von Partizipation kritisch hinterfragt werden. Die Heterogenität der Freiwilligengruppe, die dafür notwendigen methodischen Kompetenzen der Mitarbeiter*innen (insbesondere Honorarkräfte) sowie die vorhandenen beziehungsweise nicht vorhandenen Ressourcen sind hier relevante Rahmenbedingungen. Heterogenität wäre aus einem partizipationsorientierten Bildungsverständnis heraus nicht als Grenze, sondern als Bildungsgelegenheit aufzufassen. Die Formen von Partizipation bewegen sich in den Bereichen punktueller, projektorientierter, repräsentativer und offener Formen von Partizipation. Mit Sturzenhecker (2003: 30 ff.) wäre zu hinterfragen sowie von den Trägern konzeptionell zu entscheiden, welche Formen und Ebenen von Partizipation im Freiwilligendienst allgemein und im Rahmen der pädagogischen Begleitung und den Bildungsseminaren Anwendung finden sollen. Die prinzipielle Offenheit für Aushandlung, die grundsätzliche Unterstellung von Mündigkeit sowie das Begreifen von Konflikten und Widerständen als Anlass für Aushandlung und Mitwirkung sind für eine partizipatorische Bildungsarbeit grundlegend. Nach diesem Verständnis wäre zu fragen, wie der Spielraum zur Mitverantwortung und Selbstbestimmung ermöglicht und weiter vergrößert werden kann, während gleichzeitig anerkannt und berücksichtigt wird, dass die Freiwilligen unterschiedliche Vorerfahrungen und Potenziale sowie Arten und Weisen zu partizipieren mitbringen.

\section{Fazit}

Non-formale Bildung in den Freiwilligendiensten ist eng verknüpft mit dem Aspekt der Orientierung und geht gleichzeitig darüber hinaus. Der Aspekt der Orientierung zielt auf eine Unterstützung zur Bewältigung sozialer Anforderungen und Erwartungen im Sinne einer Hilfe zur Lebensbewältigung. Im Unterschied dazu lässt sich Bildung nicht auf „alltägliche Lebensbewältigung reduzieren, sondern 
[schließt] das aktive Entwerfen und Gestalten einer autonomen Lebenspraxis“ (Scherr 2002: 96) mit ein. Die derzeit politisch forcierte Verknüpfung des Bildungssystems mit dem Beschäftigungssystem strahlt auch in das Feld der Freiwilligendienste hinein. Diese Kopplung birgt die Gefahr einer einseitigen Orientierung am ökonomischen Nutzen von Bildung und den Anforderungen der Arbeits- und Wissensgesellschaft. Für das Arbeitsfeld der Jugendbildungsarbeit beschreibt Chehata (2014: 34) diese Entwicklung exemplarisch: „Die Ausbildung von sogenanntem ,Humankapital' unter dem Blickwinkel der Verwertbarkeit fragt nicht mehr danach, was Menschen lernen und können wollen, sondern nach dem, was sie können und wissen sollen." Umso zentraler erscheint eine Klärung des Bildungsverständnisses für den Bereich der Freiwilligendienste, um sich gegen Engführungen abzugrenzen und Freiräume für eine kritisch-emanzipatorische Bildungsarbeit auszuhandeln.

DerVersuch, Subjektorientierung und Partizipation als grundlegende Arbeitsprinzipien der Bildungsarbeit in den Freiwilligendiensten (wieder) stärker in den Vordergrund zu stellen, zielt darauf, Selbstbildungsprozesse zu ermöglichen, die sich von einer Logik der Ökonomisierung und Funktionalisierung abgrenzen. Dies bedeutet nicht, dass die persönliche und berufliche Orientierung sowie der Erwerb von Kompetenzen nicht auch Bestandteil von non-formaler Bildung in den Freiwilligendiensten sind. Non-formale Bildung kann aber nicht als Zulieferer für bestimmte Kompetenzbereiche, die im Vorfeld vermeintlich nicht vermittelt wurden, verstanden werden, sondern ist im Sinne eines Bildungsverständnisses, dass sich den Freiwilligen als Subjekten zuwendet, zu konzipieren. Bildung wird als Eigenaktivität des sich bildenden Subjekts verstanden und kann daher angeregt und ermöglicht, aber nicht erzwungen werden. Ziel müsste für die Bildungsarbeit entsprechend eines subjektorientierten Ansatzes demnach die Förderung von Selbstachtung, Selbstbewusstsein und Selbstbestimmung (Scherr 1997: 50 ff.) sein. An diese (konzeptionelle) Formulierung allgemeiner Wirkungsziele schließt sich die Frage an, wie diese mit Blick auf die institutionellen Rahmenbedingungen und Zielgruppen jeweils institutionell in Handlungsziele und Arbeitsweisen übersetzt werden können. Hierbei stellt sich auch die Frage, wie dieser kritisch-emanzipatorische Anspruch über die Bildungsseminare hinaus realisiert werden kann. Roth kritisiert die weitgehende Beschränkung des Partizipationsanspruches auf die Gestaltung der begleitenden Seminare und konstatiert, dass „Forderungen nach einer stärkeren Mitsprache der Freiwilligen an der Ausgestaltung ihrer Tätigkeiten“ (2017: 5) noch Mangelware sind. Somit müsse es auch um eine stärkere „Verknüpfung mit den Erfahrungen der Freiwilligen in den Organisationen und Einsätzen des FWD“ (Roth 2017: 5) gehen. In diesem Zusammenhang wäre im Zuge eines Konzeptentwicklungsprozesses mitzudenken, wie die Operationalisierung von Arbeitsprinzipien wie Subjektorientierung und Partizipation aussehen kann und in den Begleitseminaren umzusetzen wäre. 
Die Ergebnisse unserer Forschungen haben über die ideelle Idee einer kritischemanzipatorischen Bildung hinaus vielfältige Möglichkeiten der konzeptionellen Realisierung aufgezeigt. Gleichzeitig zeigt sich, dass die damit verbundenen Zielsetzungen (noch) nicht oder nur in Ansätzen Realität sind. Dennoch bieten sie eine Perspektive, damit ,sich aus der Mitwirkung in Freiwilligendiensten eine Form des bürgerschaftlichen Engagements entwickeln kann, die den ,Dienst' zugunsten von ,Engagement" und ,Partizipation“ in den Hintergrund treten lässt“ (Roth 2017: 5).

\section{Literaturverzeichnis}

Bonus, Stefanie und Stefanie Vogt (2018): Non-formale Bildung in Freiwilligendiensten. Ergebnisse aus Praxisentwicklung und Praxisforschung in kritisch-emanzipatorischer Perspektive, Baden-Baden.

Breuer, Franz (2009): Reflexive Grounded Theory. Eine Einführung für die Forschungspraxis, Wiesbaden.

Brombach, Hartmut (2014): Freiwilligendienste als Bildung, in: Journal für politische Bildung, Heft 2, S. 137-145.

Chehata, Yasmine (2014): Jugendarbeit an und in Grenzen. Kooperationen von Jugendarbeit und Schule, in: deutsche Jugend. Zeitschrift für die Jugendarbeit, Heft 1, S. 28-37.

Cloos, Peter (2010): Narrative Beobachtungsprotokolle. Konstruktion, Rekonstruktion und Verwendung, in: Auf unsicherem Terrain. Ethnographische Forschung im Kontext des Bildungs- und Sozialwesens, hrsg. von Friedericke Heinzel, Werner Thole, Peter Cloos und Stefan Köngeter, Wiesbaden, S. 181-191.

Faulstich, Peter (2013): Menschliches Lernen. Eine kritisch-pragmatische Lerntheorie, Bielefeld.

Fischer, Jörn (2011): Freiwilligendienste und ihre Wirkung - vom Nutzen des Engagements, in: Aus Politik und Zeitgeschichte, Heft 48, www.bpb.de/apuz/59669/freiwilligendiensteund-ihre-wirkung-vom-nutzen-des-engagements? $p=$ all (01.12.2017).

Jakob, Gisela (2011): Freiwilligendienste, in: Handbuch Bürgerschaftliches Engagement, hrsg. von Thomas Olk und Birger Hartnuß, Weinheim-Basel, S. 185-201.

Jakob, Gisela (2014): 50 Jahre Freiwilliges Soziales Jahr - Von einem Nischenbereich zu einer gesellschaftlichen Größe. Vortrag bei der Veranstaltung „50 Jahre Freiwilliges Soziales Jahr“, 09.09.2014, Düsseldorf.

Kelle, Udo und Susann Kluge (2010): Vom Einzelfall zum Typus. Fallvergleich und Fallkontrastierung in der qualitativen Sozialforschung, 2. Aufl., Wiesbaden.

Meuser, Michael und Ulrike Nagel (1991): ExpertInneninterviews - vielfach erprobt, wenig bedacht: ein Beitrag zur qualitativen Methodendiskussion, in: Qualitativ-empirische Sozialforschung: Konzepte, Methoden, Analysen, hrsg. von Detlef Garz und Klaus Kraimer, Opladen, http://nbn-resolving.de/ urn:nbn:de:0168-ssoar-24025 (22.02.2019).

Münchmeier, Richard, Hans-Uwe Otto und Ursula Rabe-Kleberg (Hrsg.) (2002): Bildung und Lebenskompetenz, Opladen.

Nentwig-Gesemann, Iris (2013): Die Typenbildung der dokumentarischen Methode, in: Die dokumentarische Methode und ihre Forschungspraxis. Grundlagen qualitativer Sozialforschung, hrsg. von Ralf Bohnsack, Iris Nentwig-Gesemann und Arnd-Michael Nohl, 3. Aufl., Wiesbaden, S. 295-323. 
Nugel, Martin, Peter März, Fabian Meissner und Uwe Schmidt (2014): Der Bildungsauftrag im Jugendfreiwilligendienst. Theoretisch - konzeptionelle Überlegungen zur Projektmethode als intentionale Förderung des Nicht Intentionalen, in: Voluntaris. Zeitschrift für Freiwilligendienste, Heft 1, S. 8-29.

Olk, Thomas und Ansgar Klein (2014): Bildung in Freiwilligendiensten, in: Journal für politische Bildung, Heft 2, S. 18-25.

Rauschenbach, Thomas, Hans Rudolf Leu, Sabine Lingenauber, Wolfgang Mack, Matthias Schilling, Kornelia Schneider und Ivo Züchner (2004): Non-formale und informelle Bildung im Kindes- und Jugendalter. Konzeptionelle Grundlagen für einen Nationalen Bildungsbericht, 2. Aufl., Bonn.

Rauschenbach, Thomas (2015): Bildung in Jugendfreiwilligendiensten, in: Zivil - Gesellschaft - Staat.

Freiwilligendienste zwischen staatlicher Steuerung und zivilgesellschaftlicher Gestaltung, hrsg. von Thomas Bibisidis, Jaana Eichhorn, Ansgar Klein, Christa Perabo und Susanne Rindt, Wiesbaden, S. 209-223.

Roth, Roland (2017): Engagement und Partizipation in Freiwilligendiensten. Kritische Anmerkungen zum Selbstverständnis-Papier der AG Freiwilligendienste des BBE, in: BBE - Bundesnetzwerk Bürgerschaftliches Engagement. Newsletter für Engagement und Partizipation in Deutschland, Nr. 12, S. 1-8, www.b-b-e.de/fileadmin/inhalte/aktuelles/2017/06/ newsletter-12-roth.pdf (30.11.2017).

Scherr, Albert (1997): Subjektorientierte Jugendarbeit. Eine Einführung in die Grundlagen emanzipatorischer Jugendarbeit, Weinheim-München.

Scherr, Albert (2002): Der Bildungsauftrag der Jugendarbeit. Aufgaben und Selbstverständnis im Spannungsfeld von sozialpolitischer Indienstnahme und aktueller Bildungsdebatte, in: Bildung und Lebenskompetenz, hrsg. von Richard Münchmeier, Hans-Uwe Otto und Ursula Rabe-Kleberg, Opladen, S. 93-106.

Slüter, Uwe (2008): Bildungsstandards im Freiwilligen Sozialen Jahr, in: Blätter der Wohlfahrtspflege, Heft 1, o. S.

Sturzenhecker, Benedikt (2003): Partizipation in der Offenen Jugendarbeit, in: Kiste - Bausteine für die Kinder- und Jugendbeteiligung, Entwicklung, hrsg. von BMFSFJ - Bundesministerium für Familie, Senioren, Frauen und Jugend, Berlin, https://aba-fachverband.info/ wp-content/uploads/sturzenhecker_partizipation_off_ju-1.pdf (11.04.2019).

Thimmel, Andreas (2016): Pflichtaufgabe Jugendarbeit - Junge Menschen fördern und befähigen, sich sozial und politisch zu bilden, in: Jugendhilfereport. 25 Jahre SGB VIII. Ein Gesetz im Wandel der Zeit, Köln, Heft 2, S. 20-23.

Thimmel, Andreas (2017): Bildung, in: Soziale Arbeit - Kernthemen und Problemfelder, hrsg. von Fabian Kessl, Elke Kruse, Sabine Stövesand und Werner Thole, Opladen-Toronto, S. 222-234.

Thimmel, Andreas und Hartmut Brombach (2018): Praxisforschung und Konzeptentwicklung in Freiwilligendiensten. Ein Kooperationsprojekt zwischen Internationalem Bund und dem Forschungsschwerpunkt Nonformale Bildung der TH Köln, in: Voluntaris. Zeitschrift für Freiwilligendienste, Heft 1, S. 102-113. 